\title{
NONLINEAR WAVES AND DYNAMIC PRESSURES IN RECTANGULAR TUNED LIQUID DAMPER (TLD) SIMULATION AND EXPERIMENTAL VERIFICATION
}

\author{
By Li Min SUN*, Yozo FUJINO**, Benito M. PACHECO*** and Masahiko ISOBE***
}

\begin{abstract}
Tuned Liquid Damper (TLD) utilizing the motion of shallow liquid for absorbing and dissipating the vibrational energy is studied with emphasis on liquid motion. A mathematical model based on the nonlinear shallow water wave theory is presented to describe the liquid motion in a rectangular tank. Liquid damping is evaluated semianalytically and is included in the formulation. Mechanical properties of TLD are also experimentally investigated using the shaking table. It is found that the liquid motion in TLD is strongly nonlinear and reveals a hardening-spring property even under small excitation. Good agreements between the simulation and the experimental results are shown when no breaking wave occurs. The model presented in this study is expected to serve as a tool for TLD design.

Keywords: tuned liquid damper, rectangular tank, horizontal motion, shallow wave theory, nonlinear waves, liquid damping, experiment, base shear force
\end{abstract}

\section{INTRODUCTION}

A new type of passive mechanical damper, named Tuned Liquid Damper (TLD) ${ }^{1)}$, is a tank of a certain geometry partially filled with shallow liquid and relies on the motion of liquid to dampen structural vibration (Fig. 1).

Dampers with liquid motion have been used in space satellites ${ }^{2)}$ and marine vessels ${ }^{3)}$. Modi and Welt ${ }^{4) .5)}$ carried out extensive parametric study on the nutation damper (annular tank) which is conceptually the same as TLD, and showed that it is very effective as a damper for civil engineering structures. They also investigated the energy dissipation mechanism using a nonlinear potential flow model in conjunction with boundary layer correction. Recently, Fujino, et al. ${ }^{6)}$ experimentally studied the Tuned Liquid Damper using circular tanks, in which study it was found that shallow liquid motion in a rigid tank contributed significant damping to structure with long period of oscillation. Sato ${ }^{7)}$ also studied circular TLD. Actual installations of circular TLD to tower structures in Japan are reported ${ }^{8)}$; the performance of TLD was found satisfactory. Studies on rectangular TLD are also available ${ }^{9)}$, , but an adequate mathematical model for rectangular TLD is still lacking.

A rectangular tank partially filled with water is employed as Tuned Liquid Damper in this study. To simulate the liquid motion in the tank, a mathematical model based on the nonlinear shallow water wave theory is developed. The analytical procedure is similar to the one proposed by Shimizu and Hayama ${ }^{111}$. They predicted the nonlinear sloshing of shallow liquid in rectangular storage tanks. In their study, the liquid damping was evaluated empirically from the free-oscillation experiment, whereas, the present study

* Member of JSCE, M. Eng., Graduate Student, Dept. of Civil Eng., Univ. of Tokyo (Bunkyo-ku, Tokyo 113)

** Member of JSCE, Ph. D., Asso. Prof., Eng. Res. Inst., Fac. of Eng., Univ. of Tokyo

*** Member of JSCE, Dr. Eng., Asso. Prof., Dept. of Civil Eng., Univ. of Tokyo 


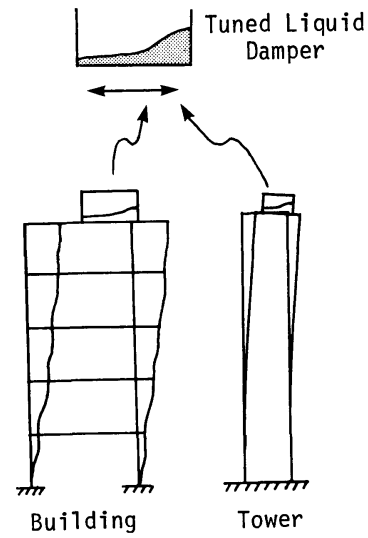

Fig. 1 Tuned Liquid Damper (TLD) installation on a building and a tower. Figures are not to scale.

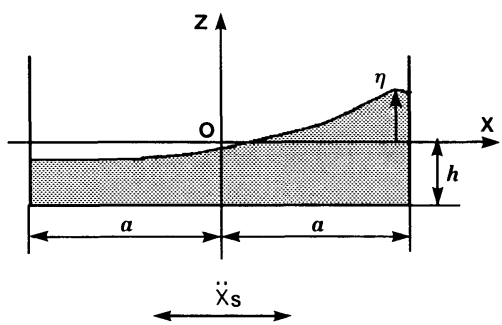

Fig. 2 Definition sketch for liquid motion in rectangular tank.

treats semi-analytically the liquid damping due to the friction at the solid boundary and the contamination at the liquid free surface. Liquid damping has a significant effect on the liquid motion near resonance.

The model thus developed can predict the liquid free surface elevation and the total horizontal pressure force due to liquid motion in the tank subjected to horizontal excitation. This model shall treat transient motion as well. Detailed experiment of TLD using the shaking table is also conducted and is used to assess the validity of the model.

\section{THEORETICAL ANALYSIS OF NONLINEAR WAVE MOTION IN RECTANGULAR TANK}

The rigid rectangular tank has a length $2 a$ and the mean liquid depth is $h$. The origin of the Cartesian coordinate system $(0-x-z)$ which is attached to the tank, is at the center of the mean liquid surface (Fig. 2). A translational motion $\ddot{x}_{s}$ (acceleration) is imposed on the tank in the $x$-direction. The following treatment is restricted to long-period oscillation and continuous surface condition (no wave breaking). The liquid particle motion is assumed to develop only in $x-z$ plane. It is also assumed that liquid is incompressible and irrotational, and that the pressure $p_{0}$ at the liquid free surface is constant.

(1) Derivation of basic equations

The full equations governing the problem are the continuity equation

$$
\frac{\partial u}{\partial x}+\frac{\partial w}{\partial z}=0 \text {, }
$$

and the equations of motion (the two-dimensional Navier Stokes equations), in which $u$ and $w$ are velocity components of liquid in the $x$ and $z$-direction, respectively. For liquid having relatively small viscosity, the effect of internal friction in the fluid is appreciable only in the boundary layer near the solid boundary (Fig. 3). From this hypothesis, the liquid flow outside the boundary layer can be treated as potential flow, and the equations of motion reduce to

$$
\begin{array}{ll}
\frac{\partial u}{\partial t}+u \frac{\partial u}{\partial x}+w \frac{\partial u}{\partial z}=-\frac{1}{\rho} \frac{\partial p}{\partial x}-\ddot{x}_{s}, & \left(-\left(h-h_{b}\right) \leqq z \leqq \eta\right) \\
\frac{\partial w}{\partial t}+u \frac{\partial w}{\partial x}+w \frac{\partial w}{\partial z}=-\frac{1}{\rho} \frac{\partial p}{\partial z}-g, & \left(-\left(h-h_{b}\right) \leqq z \leqq \eta\right)
\end{array}
$$

where $g$ denotes the gravitational acceleration, $\rho$ the fluid mass density, $p$ the pressure, and $\eta$ the surface elevation. The equations of motion for the boundary layer are described by

$$
\frac{\partial u}{\partial t}+u \frac{\partial u}{\partial x}+w \frac{\partial u}{\partial z}=-\frac{1}{\rho} \frac{\partial p}{\partial x}+\nu \frac{\partial^{2} u}{\partial z^{2}}-\ddot{x}_{s}, \quad\left(-h \leqq z \leqq-\left(h-h_{b}\right)\right)
$$




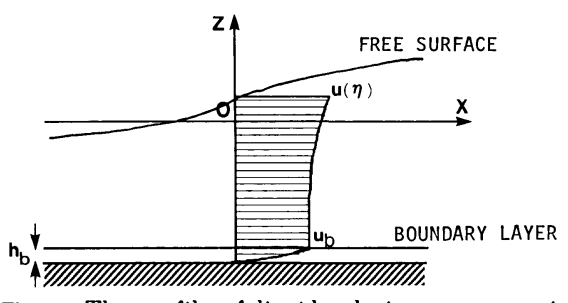

Fig. 3 The profile of liquid velocity component in $x$-direction inside and outside the boundary layer.

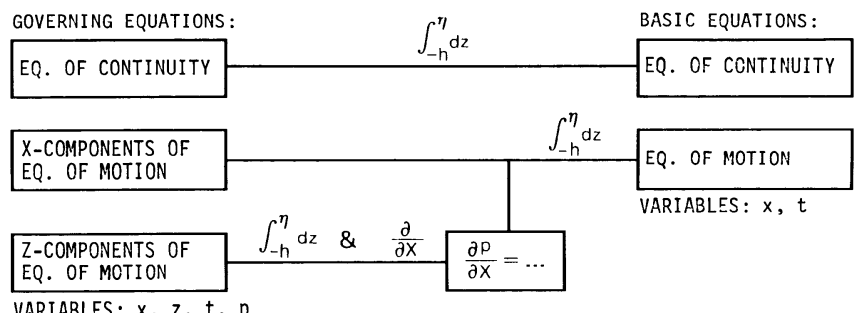

Fig. 4 Flow chart of the derivation of basic equations.

$\frac{1}{\rho} \frac{\partial p}{\partial z}=-g, \quad\left(-h \leqq z \leqq-\left(h-h_{b}\right)\right)$

where $h_{b}$ is the thickness of the boundary layer and is less than several percent of the representative length $a$ in this study ${ }^{12)}, \nu$ denotes the kinematic viscosity of liquid.

The corresponding boundary conditions are

\begin{tabular}{|c|c|}
\hline $\begin{array}{l}u=0 \\
w=0\end{array}$ & $\begin{array}{l}\text { on the wall } \quad(x= \pm a) \ldots \ldots . \\
\text { at the bottom } \quad(z=-h) \ldots\end{array}$ \\
\hline$w=\frac{D \eta}{D t}=\frac{\partial \eta}{\partial t}+u \frac{\partial \eta}{\partial x}$ & at the free surface \\
\hline$p=p_{0}=$ const & at the free surface $(z=$ \\
\hline
\end{tabular}

The velocity potential function, $\Phi$, can be defined for the irrotational part of the fluid. As an analogy to the linear wave theory, $\Phi$ is assumed as ${ }^{11)}$

$$
\Phi=F(x, t) \cosh (k(h+z)) \text {. }
$$

The vertical velocity component $w$ and its differentials can be expressed in terms of the horizontal velocity $u$ with the aid of Eq. (10). Governing equations are integrated (Fig. 4) ${ }^{12)}$ with respect to $z$ from the bottom to the free surface and the basic equations are derived as follows:

$$
\begin{aligned}
& \frac{\partial \eta}{\partial t}+h \sigma \frac{\partial(\phi u(\eta))}{\partial x}=0 \\
& \frac{\partial}{\partial t} u(\eta)+\left(1-T_{H}^{2}\right) u(\eta) \frac{\partial}{\partial x} u(\eta)+g \frac{\partial \eta}{\partial x}+g h \sigma \phi \frac{\partial^{2} \eta}{\partial x^{2}} \frac{\partial \eta}{\partial x}=\frac{\nu}{(\eta+h)} \int_{-h}^{-(h-h b)} \frac{\partial^{2} u}{\partial z^{2}} d z-\ddot{x}_{s},
\end{aligned}
$$

where $\sigma=\tanh (k h) /(k h), \phi=\tanh (k(h+\eta)) / \tanh (k h), T_{H}=\tanh (k(h+\eta)) . u(\eta)$ is the horizontal velocity component at the free surface and $k$ is the wave number. Eq. (11) is the integral of the continuity equation, and Eq. (12) has been derived from the equations of motion by eliminating the pressure $p$.

These basic equations (Eqs. (11) and (12)) are nonlinear primarily due to the free surface boundary condition (Eq. ( 8 )). The first term of right hand side in Eq. (12) comes from the bottom boundary layer, which is the integral of second term of right hand side of the equation of motion (Eq. (4)) inside the boundary layer and is interpreted as the dissipation term.

\section{(2) Damping of liquid motion}

In this formulation, it is assumed that the shear stress outside the boundary layer is negligibly small, and the dissipation term in Eq. (12) is therefore expressed as

$$
\frac{\nu}{(\eta+h)} \int_{-h}^{-\left(h-h_{b}\right)} \frac{\partial^{2} u}{\partial z^{2}} d z=-\frac{1}{(\eta+h)} \frac{1}{\rho} \tau_{b}
$$

where $\tau_{b}=\rho \nu\left(\frac{\partial u}{\partial z}\right)_{z=-h}$, is the bottom shear stress.

From the linear and laminar boundary layer theor ${ }^{13)}$ and considering sinusoidal excitation, $\tau_{b}$ is $\pi / 4$ out of phase with the horizontal velocity component of liquid just outside the boundary layer, $u_{b}$. Using the concept of equivalence of energy loss per cycle, the $\tau_{b}$ can be expressed as 


$$
\tau_{b}=\rho \frac{1}{\sqrt{2}} \sqrt{\omega \nu} u_{b},
$$

in which $1 / \sqrt{2}$ is an equivalence factor due to the phase lag $\pi / 4$, and $\omega$ is the angular frequency of the liquid motion. In the present study, since the liquid depth is shallow and the liquid motion is shallow wave, Eq. (10) gives nearly $u_{b}=u(\eta)$. Thus,

$$
\tau_{b}=\rho \frac{1}{\sqrt{2}} \sqrt{\omega \nu} u(\eta)
$$

However, the wave motion is nonlinear around the resonance, even for small amplitude excitation as will be shown later. Because of the nonlinear property, the effective phase lag between $u_{b}$ and $\tau_{b}$ is expected to be smaller than $\pi / 4$, and Eq. (15) underestimates the liquid damping even when the boundary layer is laminar. Also, when the excitation amplitude increases and the boundary layer becomes turbulent, Eq. (15) is not anymore rigorous. An attempt is made here to develop an alternate expression.

According to Jonsson's study ${ }^{(4)}$, the liquid motion inside the boundary layer depends on the Reynolds number, $R e=\left(\right.$ squared amplitude of $\left.u_{b}\right) /(\omega \nu)$. This is approximated here as

$$
R e=\frac{U^{2}(\eta)}{\omega \nu},
$$

where $U(\eta)$ is the amplitude of $u(\eta)$ at $x=0 . R e=1.26 \times 10^{4}$ is a criterion which defines the boundary layer to be laminar or turbulent. In the turbulent case, the bottom shear stress can be assumed as

$$
\tau_{b}=\frac{\rho}{2} f_{b}|u(\eta)| u(\eta)
$$

in which $f_{b}$ is the wave friction factor and is expressed in Ref. 14) as

$$
\frac{1}{4 \sqrt{f_{b}}}+2 \log _{10}\left(\frac{1}{4 \sqrt{f_{b}}}\right)=\log _{10}(R e)-1.55 \text {. }
$$

In the present problem, the $R e$ is in the range of $3 \times 10^{3}$ to $4 \times 10^{4}$, where the value of $U(\eta)$ used is computed by the numerical simulation described in the following section. For most cases, $R e$ is larger than $1.26 \times 10^{4}$, indicating that the liquid motion inside the boundary layer is turbulent. The Reynolds numbers are not much larger than $1.26 \times 10^{4}$, however. For simplicity, the following equation for laminar boundary layer is employed here, and is to be used in conjunction with Eq. (17).

$$
f_{b}=2 / \sqrt{R e}=2 \sqrt{\omega \nu} / U(\eta) \text {. }
$$

The value of $f_{b}$ is $1.16 \times 10^{2}$ by Eq. (18) and is $1.00 \times 10^{2}$ by Eq. (19) when $R e=4 \times 10^{4}$ and the underestimate by Eq. (19) is about $10 \%$.

$U(\eta)$ in Eq. (19) can be eliminated by linearizing Eq. (17). Consider the bottom shear stress $\tau_{b}$ in the form

$$
\tau_{b}=\frac{\rho}{2} f_{b} C_{e} u(\eta)
$$

where the parameter $C_{e}$ is determined by equating the energy loss per cycle by Eq. (17) to that by Eq. (20). The energy loss per cycle is

$$
W=\int_{-T / 2}^{T / 2} \tau_{b} u(\eta) d t
$$

where $T=2 \pi / \omega$, is the period of liquid motion. Substituting Eq. (17) and Eq. (20) into Eq. (21) in turn and equating the results, we obtain

$$
C_{e}=\frac{8}{3 \pi} U(\eta)
$$

Thus,

$$
\tau_{b}=\rho \frac{8}{3 \pi} \sqrt{\omega \nu} u(\eta)
$$

where the factor $8 /(3 \pi)$ replaces the factor $1 / \sqrt{2}$ in Eq. (15). The value of $\tau_{b}$ from Eq. (23) is a little 
larger than that from Eq. (15). In the present study, considering the range of Reynolds number $R e$, Eq. (23) is employed to estimate the liquid damping. It should be noted that $\tau_{b}$ expressed by Eq. (20) with Eq.

(22) is also applicable to a turbulent boundary layer by using Eq. (18).

In the derivation thus far, only the damping effect at the bottom boundary layer has been considered. Vandorn ${ }^{15)}$ reported, however, that the damping of liquid in a tank observed from experiment was larger than that computed for the bottom boundary layer. Miles ${ }^{16) .17)}$ also studied the damping of surface wave in closed basin and pointed out that the damping of liquid motion appears to be due to viscous dissipation at the solid boundary layer, viscous dissipation at the surface in consequence of surface contamination, and capillary hysteresis associated with the meniscus surrounding the free surface. Mei ${ }^{18)}$ stressed that the meniscus plays a crucial role and postulated a simple physical model to describe the damping due to the meniscus. This effect may dominate boundary-layer damping for a hydrophobic wall; but it is small for a clean, hydrophilic wall such as glass ${ }^{17)}$ or acrylic plate like the material of the TLD tank used in the present study.

Miles suggested that the dissipation term could be multiplied by $(1+(2 h / b)+S)$, where $b$ is the width of the tank, to account for the dissipation due to side wall friction and liquid surface contamination. Miles assumed that the friction of side wall boundary layer was the same as that of bottom boundary layer, $2 h / b$ is an equivalent coefficient of the damping effect per unit width due to the side wall boundary layer ${ }^{16) .17)}, S$ is a "surface contamination" factor which can vary between 0 and 2. A value of unity will be used in this study, which corresponds to the establishment of "the fully contaminated surface" 19 ).

The dissipation term with the correction of the effects of side wall and free surface is replaced by

$$
\frac{\nu}{(\eta+h)} \int_{-h}^{-(h-h b)} \frac{\partial^{2} u}{\partial z^{2}} d z=-\lambda u(\eta)
$$

where

$$
\lambda=\frac{1}{(\eta+h)} \frac{8}{3 \pi} \sqrt{\omega \nu}(1+(2 h / b)+S) .
$$

Shimizu and Hayama ${ }^{11)}$ determined the value of $\lambda$ by a free oscillation experiment. For small liquid depth ratio $\varepsilon(=h / a$, the ratio of liquid depth to half-length of the tank) of 0.1 , the value of $\lambda$ from their experiment is $0.42 \mathrm{~s} / \mathrm{cm}$. On the other hand, the value of $\lambda$ computed assuming the first natural sloshing frequency and neglecting $\eta$ in Eq. (25), is $0.38 \mathrm{~s} / \mathrm{cm}$. This indicates that Eq. (25) can predict well the damping of shallow liquid motion.

\section{(3) Numerical simulation method}

The basic equations (Eqs. (11) and (12)) are discretized with respect to $x$ into difference equations with staggered mesh and are solved numerically. The free surface waves originally possess a dispersion character, which is replaced by the dispersion relation produced by the discretization of the basic equations choosing a suitable division number $n$. The nondimensionalized wave number $k$ is taken as $\pi / 2$, since the frequency around the first natural frequency is of concern. In this paper, $n$ is calculated using ${ }^{11)}$

$n=\pi /(2 \arccos (\sqrt{(\tanh (\pi \varepsilon)) /(2 \tanh (\pi \varepsilon / 2))})) .(\varepsilon=h / a)$

After determining the division number $n$ and with the corresponding boundary condition $u(\eta)=0, \quad(x= \pm a)$

the difference basic equations are solved by Runge-Kutta-Gill method, and $u(\eta)$ and $\eta$ can be computed.

(4) Base shear force of the tank due to liquid motion

Considering hydrostatic pressure and vertical acceleration effect, the pressure $p$ can be expressed as $\frac{1}{\rho}\left(p-p_{0}\right)=g(\eta-z)-\frac{1}{k^{2}}\left(\frac{\partial^{2} u(\eta)}{\partial x \partial t}-\frac{\partial^{2} u}{\partial x \partial t}\right)$.

If $\eta$ is designated, the integration of Eq. (25) with respect to $z$ yields the horizontal total pressure $P$. Neglecting the frictions of side wall and bottom, the base shear force of the tank due to liquid motion is 
$F=P_{n}-P_{0}$,

where $P_{n}$ and $P_{0}$ are the horizontal total pressures acting on the end walls of the tank due to liquid motion and are functions of liquid free surface elevation at the end wall (Fig.5).

\section{EXPERIMENT AND COMPARISON WITH NUMERICAL SIMULATION}

\section{(1) Experimental apparatus and procedure}

In order to observe the liquid motion in TLD and to assess the validity of the model, a forced excitation experiment was carried out. A TLD tank was excited horizontally by a shaking table (Fig.6). A capacitance wave gage was used to measure liquid surface elevation near the end wall of the tank. For measuring base shear force of the TLD tank, two loadcells L1 and L2 were used to cancel the inertia force due to the TLD tank itself. Note that $M_{0}$ in Fig. 6 is a mass equivalent to the mass of the tank. Output L1-L2 is the base shear force of TLD purely due to liquid motion.

A rectangular tank with $2 a=59.0 \mathrm{~cm}$ in length (excitation direction) and $b=33.5 \mathrm{~cm}$ in width, being made of $0.5 \mathrm{~cm}$ thick acrylic plates was used (Fig. 7). The TLD tank is partially filled with plain water of $h=3.0 \mathrm{~cm}$ depth, for which the liquid depth ratio is $\varepsilon=h / a=0.1$. From the linear wave theory, the first mode frequency of liquid sloshing motion, $f_{w}$, is

$$
f_{\mathrm{w}}=\frac{1}{2 \pi}\left(\frac{\pi g}{2 a} \tanh \left(\frac{\pi h}{2 a}\right)\right)^{1 / 2}=0.458 \mathrm{~Hz} \text {. }
$$

The natural period is $T_{w}=2.18 \mathrm{sec}$, and is regarded as a typical value in tall towers. The mass of water $M_{w}$ is $5.93 \mathrm{~kg}$.

In the experiment, the TLD tank was initially quiescent and was excited sinusoidally with constant amplitude. For six amplitudes of displacement of shaking table, $A=0.1 \mathrm{~cm}, 0.25 \mathrm{~cm}, 0.5 \mathrm{~cm}, 1.0 \mathrm{~cm}$, $2.0 \mathrm{~cm}$ and $4.0 \mathrm{~cm}$, the excitation frequency $f$ was varied in the range of $0.8<f / f_{w}<1.5$. The experimental cases are shown in Table 1.

The quantities measured in the experiment are : 1) displacement of shaking table, $x_{s} ; 2$ ) liquid free surface elevation near the end wall, $\eta_{0}$; and 3) base shear force of TLD model due to liquid motion, $F$. The analog signals are converted to digital ones, and they are processed by a micro-computer.

\section{( 2 ) Experimental results}

Fig. 8 shows sample steady-state time histories of displacement of shaking table, $x_{s}$; liquid free surface elevation, $\eta_{0}$; and base shear force, $F$. The wave forms of these time histories vary as either the excitation frequency or the base amplitude varies. When the wave amplitude is small (Fig. $8(\mathrm{a})$ ), the time histories of $\eta_{0}$ and $F$ are in the sinusoidal form. For large wave amplitude, however, the time histories become nonsinusoidal (Fig. $8(\mathrm{~b}),(\mathrm{c})$, and (d)). At a certain excitation frequency, higher frequency

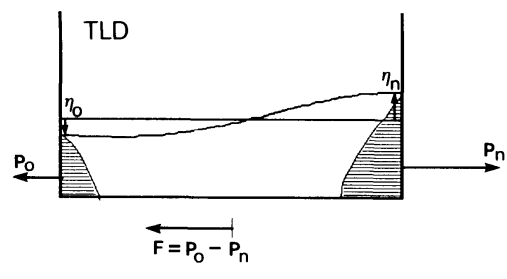

Fig. 5 Base shear force of tank due to liquid motion.

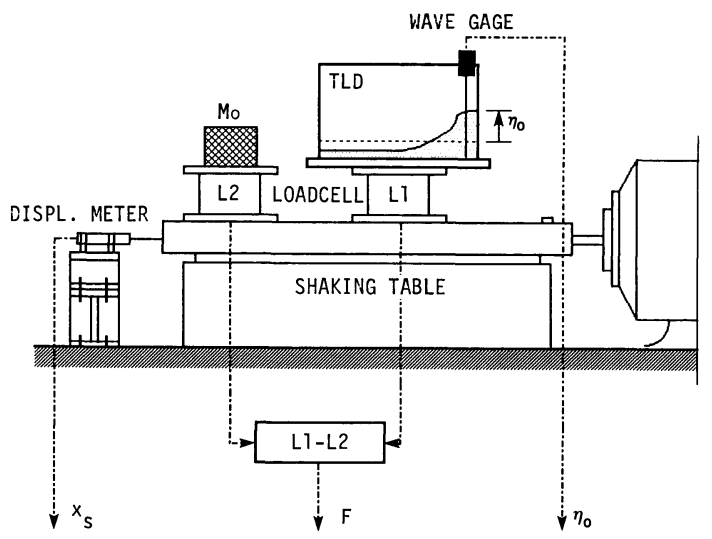

Fig. 6 The experimental apparatus. 


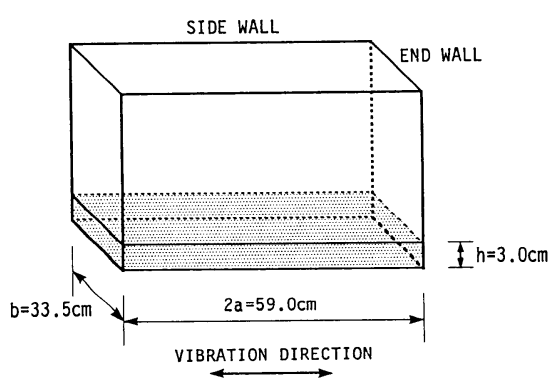

Table 1 The Experimental Cases.

Fig. 7 TLD tank.

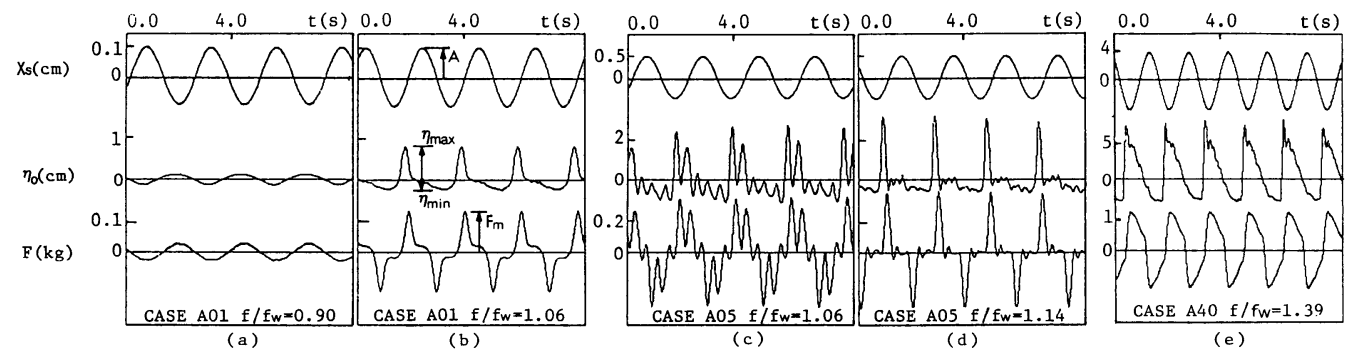

Fig. 8 Sample time histories of displacement of shaking table, $x_{s}$, liquid surface elevation near the end wall, $\eta_{0}$, and base shear force, $F$.

response are observed (for example, Fig. 8(c)). This can be attributed to nonlinearity of the liquid motion, namely internal resonance ${ }^{20)}$. For a larger excitation, wave form is distorted apparently because of wave breaking (Fig. 8(e)).

Several nondimensionalized parameters are defined as follows for the presentation of results:

During one cycle of liquid motion, liquid free surface elevation, $\eta_{0}$, has a maximum value $\eta_{\max }$ (wave crest) and a minimum value $\eta_{\min }$ (wave trough) (Fig. 8). The nondimensionalized parameters $\eta_{\max }$ and $\eta_{\min }$ are defined as

$$
\eta_{\max }^{\prime}=\eta_{\max } / h ; \quad \eta_{\min }^{\prime}=\eta_{\min } / h
$$

where $h$ is the liquid depth.

Under the sinusoidal excitation, base shear force $F(t)$ has the same amplitude $F_{m}$ in either positive or negative direction (Fig. 8). The maximum inertia force of liquid as a solid mass under the sinusoidal excitation is used to nondimensionalize $F_{m}$ :

$F_{m}^{\prime}=F_{m} /\left(M_{w} \omega^{2} A\right)$

The shaking table inputs energy into the TLD system and the TLD itself dissipates energy due to liquid damping. When the TLD is at steady state, it means that in each cycle the energy input into the TLD system equals the energy dissipation inside the TLD. The energy input into the TLD can be calculated from the base shear force $F$ and the displacement of shaking table $x_{s}$, which are both functions of time. Thus the energy dissipation per cycle, $\Delta E$, can be calculated by

$$
\Delta E=\int_{t}^{t+T} F(t) d x_{s}(t)
$$

where $T$ is the period of excitation.

Fig. 9 shows examples of the force-displacement diagrams in one cycle. The maximum base shear force does not increase in proportion with the base amplitude $A$. The energy dissipation per cycle can be expressed by the area of $F-x_{s}$ loop presented in Fig. 9. The $F-x_{s}$ diagram is not ellipsoid $(A=0.5 \mathrm{~cm}$ in Fig. 9) because $F$ is not sinusoidal (Fig. $8(\mathrm{c})$ ). When the amplitude $A$ is $2.0 \mathrm{~cm}$, the $F-x_{s}$ loop is close to a rectangular shape, indicating that the system is of a friction-controlled type apparently due to the 
occurrence of wave breaking.

$\Delta E$ is nondimensionalized as follows :

$$
\Delta E^{\prime}=\Delta E /\left(\frac{1}{2} M_{w}(\omega A)^{2}\right) \text {. }
$$

It should be noted that $1 / 2 M_{w}(\omega A)^{2}$ is not the energy of liquid motion but just a reference value.

\section{( 3 ) Comparison and discussion}

For numerical simulation, the motion of TLD is assumed to be quiescent at $t=0$. The time increment was $1 / 60$ of the excitation period of shaking table. The computation was carried on until 80 periods,

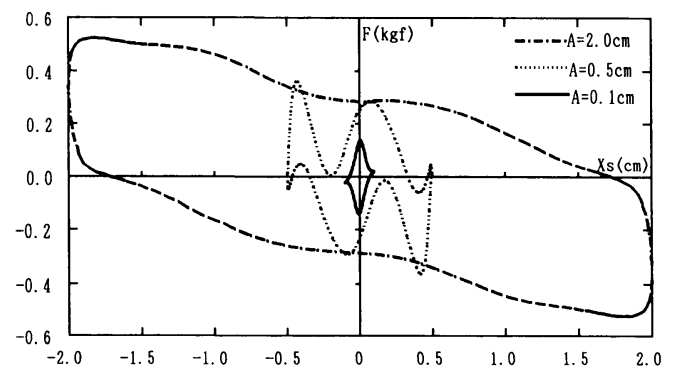

Fig. 9 Diagrams between base shear force $F$ and displacement of shaking table $x_{s}\left(f / f_{\mathrm{W}}=1.06\right)$. where liquid motion was regarded to be already at steady state.

The nondimensionalized quantities, $\eta_{\max }^{\prime}, \eta_{\min }^{\prime}$ (surface elevation), $F_{m}^{\prime}$ (maximum base shear force) and $\Delta E^{\prime}$ (energy loss in TLD per cycle) are plotted in Fig. 10 for the frequency range of $0.8<f / f_{w}<1$. 3 . In the experiment, when the input amplitude was larger than $0.5 \mathrm{~cm}$ (Cases A 10, A 20 and A 40), splash near the end wall and turbulent wave on the free surface were observed, indicating that breaking wave already occurred. Hence, the computations were made only for Cases A 01, A 025, A 05 and A 10.

The comparisons of nonlinear simulations using Eq. (15) (broken line …..) and Eq. (23) (solid line - ) are shown in Figs. 10 (a) - (c). It is found that the difference is small. For very small $R e$ where the wave motion is linear, Eq. (15) should be more suitable and more reasonable. However, for the discussion of the present cases, we use Eq. (23). For other cases where $R e$ is much larger than $1.26 \times 10^{4}$, the damping may be underestimated even by Eq. (23).

For the relatively small amplitude excitation (Cases A 01, A 025 and A 05), the simulation can predict well the experimental results $\left(\eta_{\max }^{\prime}, \eta_{\min }^{\prime}, F_{m}^{\prime}\right.$ and $\left.\Delta E^{\prime}\right)$. For Case A 10 (Fig. $10(\mathrm{~d})$ ), however, the simulation results do not agree with the experimental ones anymore. The results indicate that the model used here is valid as far as the continuous free surface condition is satisfied, i. e., no wave breaking.

The experiments and the simulations indicate that the liquid motion possesses hardening-type nonlinearity ${ }^{21)}$. For example, $\eta_{\max }^{\prime}$ jumps suddenly at a certain frequency ratio. This jump frequency ratio is larger than 1.00, confirming that the nonlinearity of liquid motion is hardening-spring type. As the input amplitude increases, the jump frequency ratio becomes higher, indicating that the nonlinearity becomes stronger. The jump frequency ratio for Case A $01(A=1.0 \mathrm{~cm})$ is about 1.1 and increases to about 1.4 for Case A $20(A=2.0 \mathrm{~cm})$. When input amplitude is $4.0 \mathrm{~cm}$ (Case A 40) (Fig. 10(f)), $\eta_{\max }^{\prime}$ does not jump before $f / f_{\mathrm{W}}=1$.5. Local peaks of $\eta_{\max }^{\prime}$ (or of $F_{m}^{\prime}$ or $\Delta E$ ) correspond to appearance of higher modes as seen for example in Fig. 8(c) for Case A 05.

Fig. 11 presents sample time histories of surface elevation, $\eta_{0}$, and base shear force, $F$, for Case A 025. The response forms vary as the excitation frequency changes, due to nonlinearity of the liquid motions as discussed above. For example, there are two waves in one cycle at $f / f_{w}=1.001$, and three waves can be observed at $f / f_{W}=0.951$. Numerical simulations corresponding to Case A 025 are also shown in Fig. 11 for comparison. Good agreement can be seen even in the transient interval. The theory used here is satisfactory in accounting for even the nonlinearity which induces higher modes of liquid motion.

Fig. 12 shows examples of force-displacement diagrams, also for Case A 025. One can find that the simulation results agree well with the experiment. The model can predict the phase lag between the excitation displacement and the base shear force.

For large excitation amplitude (Fig. 10 (f), Case A 40), $F_{m}^{\prime}$ and $\Delta E^{\prime}$ are almost flat over a wide range of frequency, implying that TLD becomes a highly-damped system. The efficiency of TLD as a damper may 

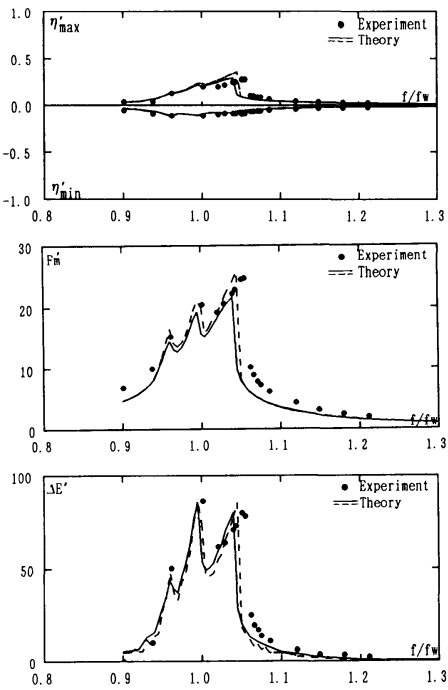

(a) Case A 01
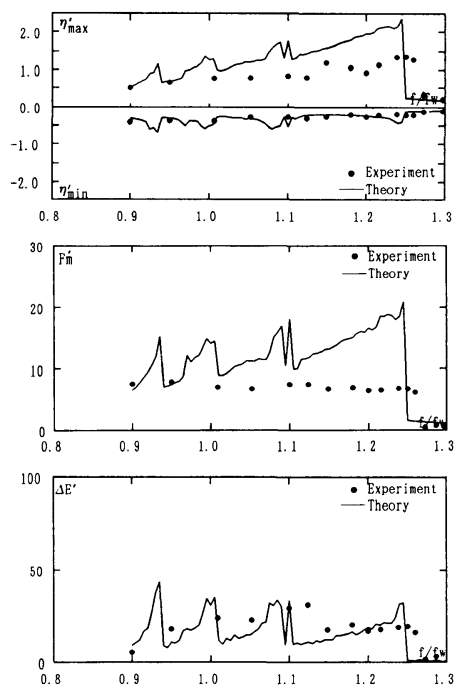

(d) Case A 10
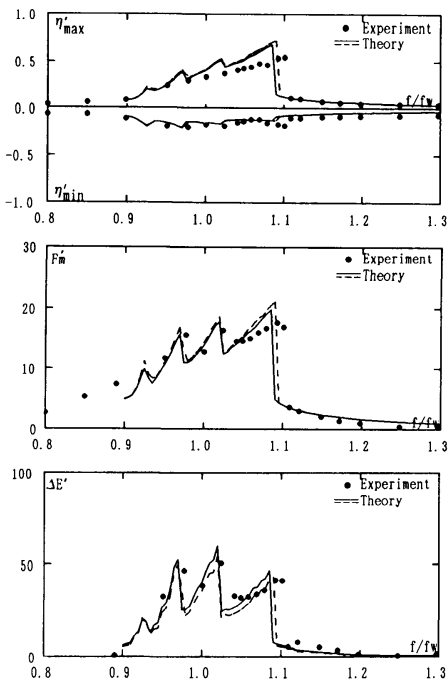

(b) Case A 025
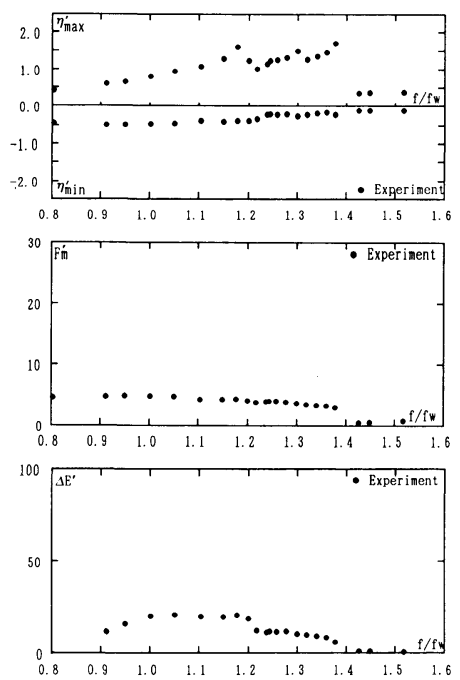

(e) Case A 20
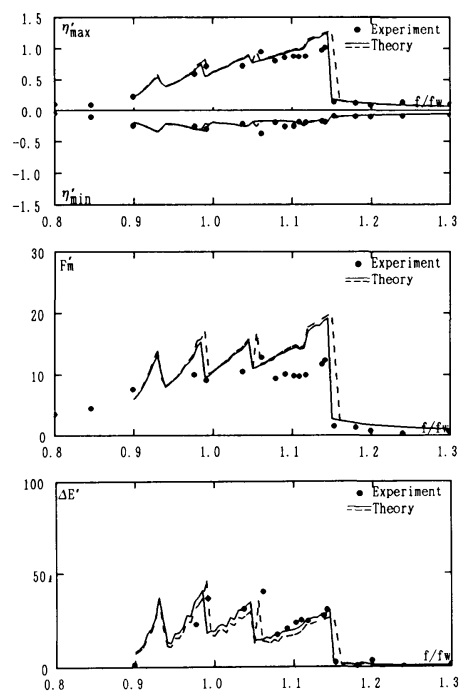

(c) Case A 05
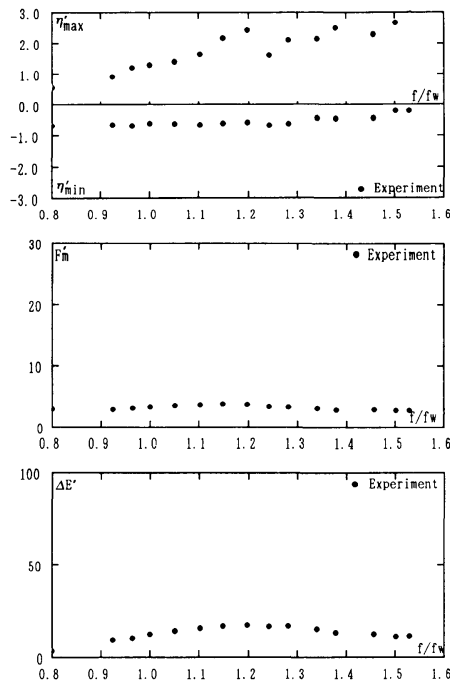

(f) Case A 40

Fig. 10 Frequency responses of $\eta_{\max }^{\prime}$ and $\eta_{\min }^{\prime}(\mathrm{Eq} .31), F_{m}^{\prime}(\mathrm{Eq} .32)$, and $\Delta E^{\prime}$ (Eq. 34) (— Theory using Eq. (23); ….. Theory using Eq. (15))

be decreased when the vibration amplitude of the structure with TLD is so large that wave breaking occurs. Similarly, a linear tuned mass damper loses its efficiency when the damping in TMD is too high. On the other hand, it should be understood from the flatness of the frequency-response curve that the tuning of TLD to the natural frequency of the structure is not so important for large amplitude excitation. These trends at large amplitude are consistent with the findings obtained in the free-oscillation experiment ${ }^{6}$.

\section{CONCLUDING REMARKS}

The liquid motion in the TLD tank has been investigated both by experiment and by theoretical analysis. 


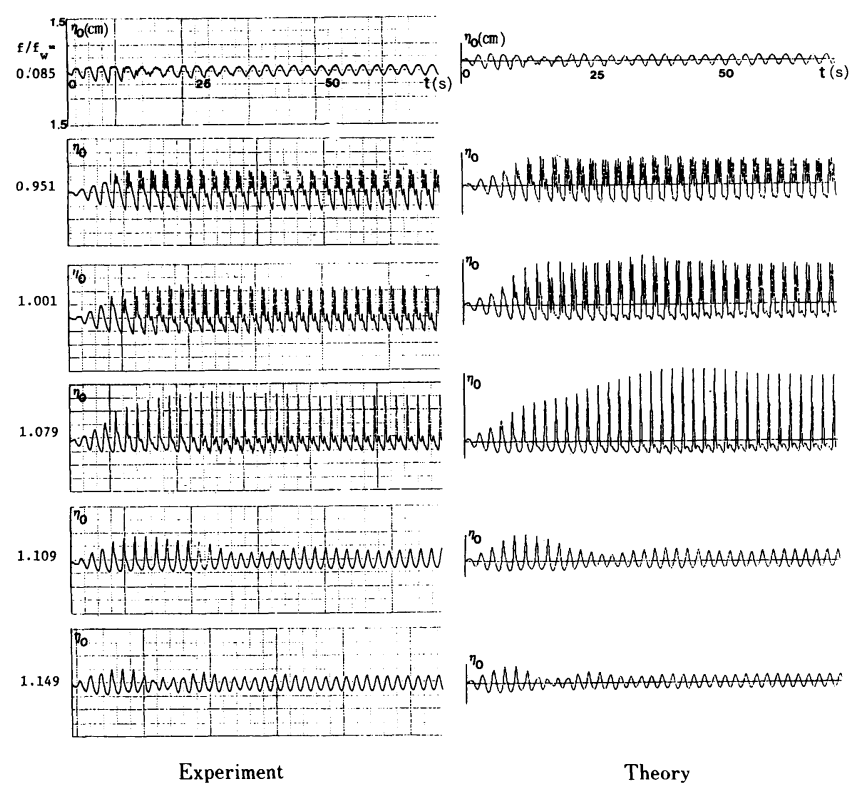

(a) wave surface elevation $\eta_{0}$

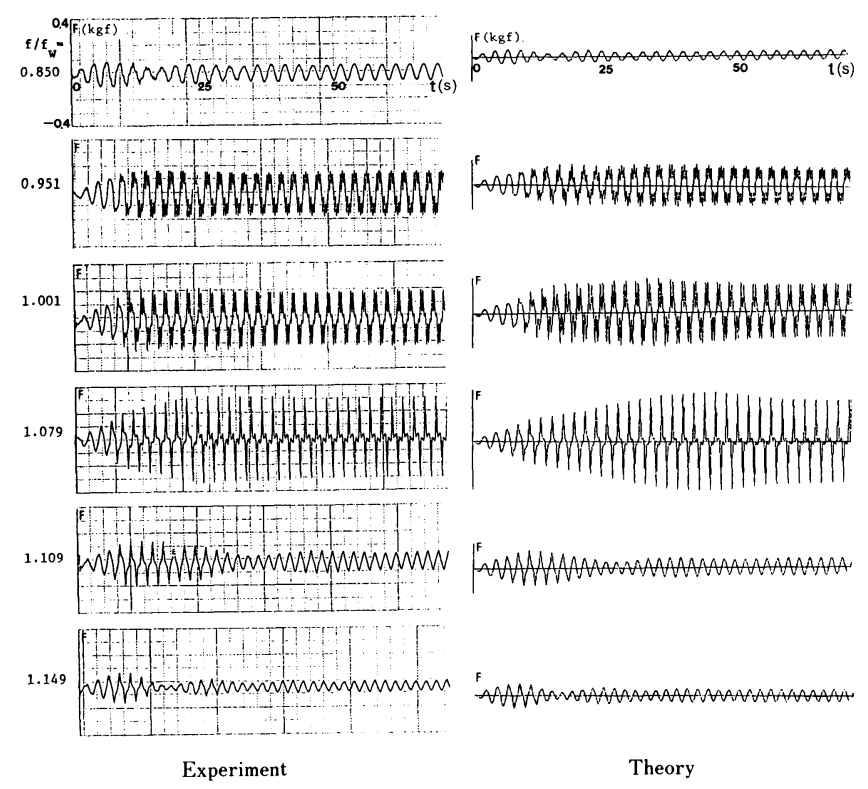

(b) base shear force $F$
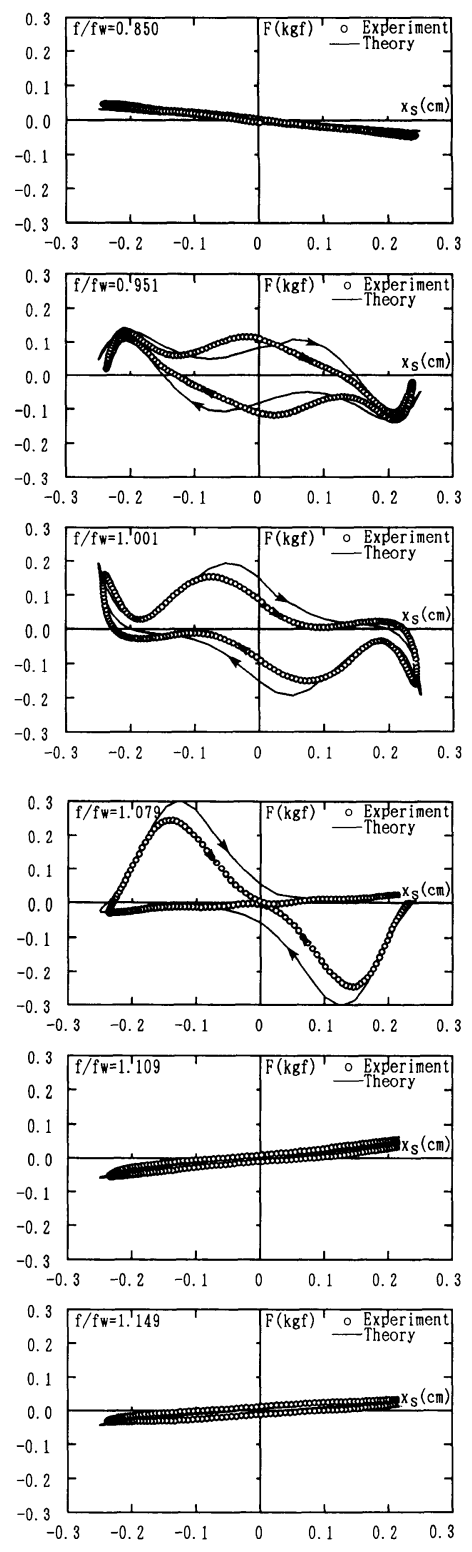

Fig. 12 The energy dissipation loops (Case A 025).

Fig. 11 The time histories of $\eta_{0}$ and $F$ (Case A 025).

The mathematical model developed in this study has been found satisfactory when the oscillation amplitude of TLD is not too large and no breaking wave occurs yet. In the range of the present study, for which liquid depth is rather shallow, the liquid motion possesses a strong nonlinearity of hardening-spring type. The nonlinearity of liquid motion also induces the higher modes through internal resonance. These higher modes also can be simulated by the present model.

Mild turbulence in the boundary layer has been considered in theoretically estimating the liquid damping. 
Incorporating this damping term, the model has proved to be capable of simulating even the transient motion. Although there still is limitation to the applicability, the model can be conveniently used to study interaction of TLD and structure ${ }^{22)}$ for the purpose of optimizing the TLD design.

The liquid motion is more complicated when breaking wave occurs. The present model does not account for this, and development of a model which includes the breaking wave effect remains for future study. An engineering approach is given in Ref.23).

\section{ACKNOWLEDGEMENTS}

The authors would like to express their deep gratitude to Mr. Piyawat Chaiseri, doctoral student at the University of Tokyo, for his help in the experiment. Encouragement from Prof. M. Ito at the Univ. of Tokyo through this study is also fully acknowledged. The authors would also like to thank Mr. K. Fujii and Mr. T. Sato, Shimizu Corporation, for allowing the use of the shaking table facility and giving suggestions during the experiment. This research was supported partially by a Grant-in-Aid from the Japan Ministry of Education, Science, and Culture, and the Joint Research Program with Shimizu Corp.

\section{REFERENCES}

1) Chaiseri, P. : Damper for Tower-like Structure Utilizing Liquid Motion as Energy Dissipator, Master Thesis, Dept. of Civil Engg. Univ. of Tokyo, Japan, 1987.

2) Bhuta, P. G. and Koval, L. R. : A Viscous Ring Damper for a Freely Precessing Satellite, International Journal of Mechanical Science, . Vol. 8, pp. 383-395, 1966.

3) Watanabe, S. : Methods of Vibration Reduction, Proc. Japan Naval. Arch. Soc. Symp., pp. 156-189, 1969 (In Japanese) .

4) Modi, V. J. and Welt, F. : Vibration Control Using Nutation Dampers, Proc. International Conference on Flow Induced Vibrations, England, pp. 369-376, 1987.

5) Welt, F. : A Study of Nutation Dampers with Application to Wind Induced Oscillations, Ph. D. Thesis, The University of British Columbia, 204 pp. , 1988.

6) Fujino, Y. et al. : Parametric Studies on Tuned Liquid Damper (TLD) Using Circular Containers By Free-oscillation Experiment, Structural Eng./Earthquake Eng. JSCE, No. 398, 1988.

7) Sato, T. : Tuned Sloshing Damper, Japan J. Wind Eng., Vol. 32, pp. 67-68, 1987 (In Japanese).

8) Tamura, Y. and Fujii, K. et al. : Wind-Induced Vibration of Tall Towers and Practical Applications of Tuned Sloshing Damper, Proc. Symposium/Workshop on Serviceability of Buildings, Canada, pp. 228-241, 1988.

9) Matsuura, Y. et al. : On a Mean to Reduce Excited-vibration with the Sloshing in a Tank, Proc. Japan Naval. Arch. Soc., No. 160, pp. 424-432, 1986 (In Japanese).

10) Miyata, T. et al. : Suppression of Tower-like Structures Vibration by Damping Effect of Sloshing Water Contained, J. Struct Eng., JSCE, Vol. 34 A, pp.618-625, 1988 (In Japanese).

11) Shimizu, T. and Hayama, S. : Nonlinear Response of Sloshing Based on the Shallow Water Wave Theory, JSME International Journal, Vol.30, No.263, pp.806-813 (or JSME, Vol.53 (486), pp. 357-363, 1987 (In Japanese)).

12) Sun, L. M. : Simulation of Nonlinear Waves in Rectangular Tuned Liquid Damper (TLD), Master Thesis, Dept. of Civil Engg. Univ. of Tokyo, Japan, 1988.

13) Lamb, H. : Hydrodynamics, Cambridge Univ. Press, pp. 619-621, 1932.

14) Jonsson, I. G. : Wave Boundary Layer and Friction Factors, Proc. 10 th CCE, ASCE, pp. 127-148, 1966.

15) Vandorn, W. G. : Boundary Dissipation of Oscillatory Waves, Journal of Fluid Mechanics, Vol. 24, part 4, pp. 769-779, 1966.

16) Miles, J. W. : Surface Wave Damping in Closed Basins, Proceedings, Royal Society of London, A 297, pp. 459-475, 1967.

17) Miles, J. W. : Resonantly Forced, Nonlinear Gravity Waves in a Shallow Rectangular Tank, Wave Motion, Elsevier Science Publishers, B. V. (North Holland), 7, pp. 291-297, 1985.

18) Mei, C. C. : The Applied Dynamics of Ocean Surface Waves, John Wiley \& Sons, pp. 384-481, 1983.

19) Lepelletier, T. G. and Raichlen, F. : Nonlinear Oscillations in Rectangular Tanks, Journal of Engineering Mechanics, 114 ( 1 ), ASCE, pp. 1-23, 1988.

20) Nayfeh, A.H. and Mook, D. T. : Nonlinear Oscillations, John Wiley \& Sons, pp. 6-20, 402-424, 1979.

21) Hayama, S. et al. : Nonlinear Response of Sloshing in Rectangular Tank, JSME (c), Vol. 49, No. 437, pp. 22-33, 1983 (In Japanese).

22) Chaiseri, P. et al. : Interaction of Tuned Liquid Damper (TLD) and Structure - Theory, Experimental Verification and Application-, Structural Eng./Earthquake Eng., Vol.6, No.2, JSCE, 1989. 
23) Pacheco, B. M. and Fujino, Y. : Mass and Dashpot Representation of Non-linear Passive Mechanical Dampers, Proc. 44 th JSCE Annual Meeting, Vol. 1, 2 pp., 1989.

(Received September 26 1988) 